\title{
FOURIER SERIES OF FUNCTIONS OF $\Lambda$-BOUNDED VARIATION
}

\author{
DANIEL WATERMAN ${ }^{1}$
}

\begin{abstract}
It is shown that the Fourier coefficients of functions of $\Lambda$ bounded variation, $\Lambda=\left\{\lambda_{n}\right\}$, are $O\left(\lambda_{n} / n\right)$. This was known for $\lambda_{n}=n^{\beta+1}$, $-1<\beta<0$. The classes $L$ and HBV are shown to be complementary, but $L$ and $\Lambda \mathrm{BV}$ are not complementary if $\Lambda \mathrm{BV}$ is not contained in HBV. The partial sums of the Fourier series of a function of harmonic bounded variation are shown to be uniformly bounded and a theorem analogous to that of Dirichlet is shown for this class of functions without recourse to the Lebesgue test.
\end{abstract}

We have shown elsewhere that functions of harmonic bounded variation (HBV) satisfy the Lebesgue test for convergence of their Fourier series, but if a class of functions of $\Lambda$-bounded variation $(\Lambda \mathrm{BV})$ is not properly contained in $\mathrm{HBV}$, it contains functions whose Fourier series diverge [1]. We have also shown that Fourier series of functions of class $\left\{n^{\beta+1}\right\}-B V,-1<\beta<0$, are $(C, \beta)$ bounded, implying that the Fourier coefficients are $O\left(n^{\beta}\right)$ [2].

Here we shall estimate the Fourier coefficients of functions in $\Lambda \mathrm{BV}$. Without recourse to the Lebesgue test, we shall prove a theorem for functions of HBV analogous to that of Dirichlet and also show that the partial sums of the Fourier series of an HBV function are uniformly bounded. From this one can conclude that $L$ and HBV are complementary classes, i.e., Parseval's formula holds (with ordinary convergence) for $f \in L$ and $g \in \mathrm{HBV}$. We shall see that $L$ and $\Lambda \mathrm{BV}$ are not complementary if $\Lambda \mathrm{BV}$ is not a subclass of HBV.

1. Definitions and results. Let $f$ be a real function on an interval $[a, b]$, $\Lambda=\left\{\lambda_{n}\right\}$ a nondecreasing sequence of positive numbers such that $\sum 1 / \lambda_{n}$ diverges, and $\left\{I_{n}\right\}$ a sequence of nonoverlapping intervals $I_{n}=\left[a_{n}, b_{n}\right] \subset$ $[a, b]$. The function $f$ is said to be of $\Lambda$-bounded variation $(\Lambda \mathrm{BV})$ if $\Sigma \mid f\left(a_{n}\right)-$ $f\left(b_{n}\right) \mid / \lambda_{n}$ converges for every choice of $\left\{I_{n}\right\}$. The supremum of these sums is called the $\Lambda$-variation of $f$, denoted by $V_{\Lambda}(f ;[a, b])$. When $\Lambda=\{n\}$, the class is referred to as the functions of harmonic bounded variation (HBV).

We shall suppose that $[a, b]=[0,2 \pi]$ and our functions have period $2 \pi$. Two classes of functions, $K$ and $K_{1}$, are said to be complementary [4, p. 157] if $f \in K$ and $g \in K_{1}$ implies

$$
\frac{1}{\pi} \int_{0}^{2 \pi} f g d x=\frac{1}{2} a_{0} a_{0}^{\prime}+\sum_{1}^{\infty}\left(a_{k} a_{k}^{\prime}+b_{k} b_{k}^{\prime}\right)
$$

Received by the editors April 14, 1978.

AMS (MOS) subject classifications (1970). Primary 42A28; Secondary 26A45.

'Supported in part by NSF grant MCS77-00840. 
where $a_{k}, b_{k}$ are the Fourier coefficients of $f$ and $a_{k}^{\prime}, b_{k}^{\prime}$ are the Fourier coefficients of $g$. Here we suppose that the series on the right converges.

We shall prove the following results.

THEOREM 1. If $f \in \Lambda \mathrm{BV}$, then the Fourier coefficients of $f$ are $O\left(\lambda_{n} / n\right)$.

THeOREM 2. The classes $L$ and $\mathrm{HBV}$ are complementary. If $\Lambda \mathrm{BV}$ is not contained in $\mathrm{HBV}$, then $L$ and $\Lambda \mathrm{BV}$ are not complementary.

TheOREM 3. If $f \in \mathrm{HBV}$, then the partial sums of its Fourier series are uniformly bounded. The series converges everywhere and converges uniformly on closed intervals of points of continuity.

2. Proof of Theorem 1. Suppose we consider $b_{n}$. We have

$$
\begin{aligned}
\pi b_{n} & =\int_{0}^{2 \pi} f(t) \sin n t d t=\frac{1}{n} \sum_{0}^{2 n-1}(-1)^{k} \int_{0}^{\pi} f\left(\frac{t+k \pi}{n}\right) \sin t d t \\
& =\frac{1}{n} \int_{0}^{\pi} \sum_{1}^{2 n-1}\left[f\left(\frac{t+(k-1) \pi}{n}\right)-f\left(\frac{t+k \pi}{n}\right)\right] \sin t d t,
\end{aligned}
$$

where $^{*}$ denotes summation over odd indices. Hence

$$
\begin{aligned}
\pi\left|b_{n}\right| & <\frac{1}{n} \int_{0}^{\pi} \sum_{1}^{2 n-1}\left|f\left(\frac{t+(k-1) \pi}{n}\right)-f\left(\frac{t+k \pi}{n}\right)\right| d t \\
& =\frac{1}{n} \int_{0}^{\pi} \sum^{*} \frac{1}{\lambda_{k}}|\cdots| \lambda_{k} d t .
\end{aligned}
$$

Applying Abel's transformation, we see that this expression is $O\left(\lambda_{n} / n\right)$.

3. Proof of Theorem 2. Let us suppose that $f \in L, g \in \mathrm{HBV}$, and $S_{n}(g, x)$ is the $n$th partial sum of the Fourier series of $g$. Then

$$
\begin{aligned}
\Delta_{n} & =\left|\frac{1}{\pi} \int_{0}^{2 \pi} f g d x-\left[\frac{1}{2} a_{0} a_{0}^{\prime}+\sum_{1}^{n}\left(a_{k} a_{k}^{\prime}+b_{k} b_{k}^{\prime}\right)\right]\right| \\
& =\frac{1}{\pi}\left|\int_{0}^{2 \pi}\left(g-S_{n}(g)\right) f d x\right| .
\end{aligned}
$$

If we assume Theorem 3 , then $S_{n}(g) \rightarrow g$ everywhere and $S_{n}(g)$ is uniformly bounded. Applying the dominated convergence theorem, we have $\Delta_{n} \rightarrow 0$. Thus $L$ and $\mathrm{HBV}$ are complementary.

We now assume that $\Lambda \mathrm{BV}$ is not contained in $\mathrm{HBV}$ and show that, under this assumption, there is an $f_{0} \in L$ and a $g_{0} \in \Lambda \mathrm{BV}$ such that $\left\{\int_{0}^{2 \pi} f_{0} S_{n}\left(g_{0}\right) d x\right\}$ is a divergent sequence.

Our assumption is equivalent to the existence of a nonincreasing sequence of positive numbers $a_{n}$ such that $\sum a_{n} / \lambda_{n}$ converges, but $\sum a_{n} / n$ diverges. Let $g_{n}(x)$ be a function of period $2 \pi$ defined in $[0,2 \pi]$ to be $a_{i}$ for $(2 i-2) \pi<(n$ $\left.+\frac{1}{2}\right) x<(2 i-1) \pi, i=1, \ldots, n+1$, and 0 elsewhere. Clearly $g_{n} \in \Lambda \mathrm{BV}$. Now $\Lambda \mathrm{BV}$ is a Banach space with norm [3] 


$$
\|g\|_{\Lambda}=|g(0)|+V_{\Lambda}(g ;[0,2 \pi]) \text {. }
$$

We have

$$
V_{\Lambda}\left(g_{n} ;[0,2 \pi]\right)=\sum_{1}^{n+1} a_{i}\left(1 / \lambda_{2 i-1}+1 / \lambda_{2 i}\right) \leqslant 2 \sum_{1}^{n+1} a_{i} / \lambda_{i} .
$$

Hence

$$
\left\|g_{n}\right\|_{\Lambda} \leqslant 2 \sum_{1}^{\infty} a_{n} / \lambda_{n}=C<\infty
$$

for every $n$. Now

$$
\begin{aligned}
\sup _{x}\left|S_{n}\left(g_{n}, x\right)\right| & \geqslant\left|S_{n}\left(g_{n}, 0\right)\right|=\frac{1}{\pi}\left|\int_{0}^{2 \pi} g_{n}(t) \frac{\sin \left(n+\frac{1}{2}\right) t}{\sin \frac{1}{2} t} d t\right| \\
& =\frac{1}{\pi}\left|\sum_{0}^{2 n} \frac{(-1)^{k}}{n+\frac{1}{2}} \int_{0}^{\pi} g_{n}\left(\frac{t+k \pi}{n+\frac{1}{2}}\right) \frac{\sin t}{\sin ((t+k \pi) /(2 n+1))} d t\right| \\
& \geqslant \frac{2}{\pi} \sum_{1}^{n+1} a_{i} \int_{0}^{\pi} \frac{\sin t}{t+(2 i-2) \pi} d t \\
& \geqslant \frac{4}{\pi^{2}} \sum_{1}^{n+1} a_{i} /(2 i-1)>\frac{2}{\pi^{2}} \sum_{1}^{n+1} a_{i} / i,
\end{aligned}
$$

implying that $\sup _{x}\left|S_{n}\left(g_{n}, x\right)\right| \neq O(1)$.

Let $P_{n}(f)$ be the continuous linear functional on $L$ defined by

$$
P_{n}(f)=\int_{0}^{2 \pi} f S_{n}\left(g_{n}\right) d x
$$

Then

$$
\left\|P_{n}\right\|=\sup _{x}\left|S_{n}\left(g_{n}, x\right)\right| \neq O(1)
$$

implying that there is an $f_{0} \in L$ such that

$$
P_{n}\left(f_{0}\right) \neq O(1) \text {. }
$$

Let $Q_{n}(g)$ be the continuous linear functional on $\Lambda \mathrm{BV}$ defined by

$$
Q_{n}(g)=\int_{0}^{2 \pi} f_{0} S_{n}(g) d x
$$

Then

$$
\left\|Q_{n}\right\|>\left|Q_{n}\left(g_{n}\right)\right| /\left\|g_{n}\right\|_{\Lambda} \geqslant\left|P_{n}\left(f_{0}\right)\right| / C \neq O(1) .
$$

Hence there is a $g_{0}$ in $\Lambda \mathrm{BV}$ such that $Q_{n}\left(g_{0}\right) \neq O(1)$, implying that $\left\{\int_{0}^{2 \pi} f_{0} S_{n}\left(g_{0}\right) d x\right\}$ diverges.

4. Proof of Theorem 3. Suppose $f \in \mathrm{HBV}$. If $S_{n}(x)$ denotes the $n$th partial sum of the Fourier series of $f$, then for any $\delta>0$,

$$
S_{n}(x)-f(x)=\frac{1}{\pi} \int_{0}^{\delta}(f(x+t)+f(x-t)-2 f(x)) \frac{\sin n t}{t} d t+o(1)
$$


uniformly in $x$. Since $f(x+0)$ and $f(x-0)$ exist at each point, we may assume that $f(x)=\frac{1}{2}[f(x+0)+f(x-0)]$ for each $x$. Therefore we may write the integral above as

$$
\int_{0}^{\delta}(f(x+t)-f(x+0)) \frac{\sin n t}{t} d t+\int_{0}^{\delta}(f(x-t)-f(x-0)) \frac{\sin n t}{t} d t .
$$

We consider only the first of these. The other may be treated in an analogous manner.

Letting $h(t)=f(x+t)-f(x+0)$, we see that

$$
\left|\int_{0}^{\pi / n} h(t) \frac{\sin n t}{t} d t\right|<\pi \sup _{0<t<\pi / n}|h(t)|=o(1)
$$

for each $x$ and uniformly on closed intervals of points of continuity. This expression is also uniformly bounded. Then

$$
\begin{aligned}
\int_{\pi / n}^{\delta} h(t) \frac{\sin n t}{t} d t & =\sum_{1}^{N} \int_{k \pi / n}^{(k+1) \pi / n} h(t) \frac{\sin n t}{t} d t+\int_{(N+1) \pi / n}^{\delta} \cdots \\
& =I_{1}+I_{2}
\end{aligned}
$$

where $N+1=[n \delta / \pi]$. Clearly $I_{2}=o(1)$ uniformly in $x$ and

$$
I_{1}=\int_{0}^{\pi} \sum_{1}^{N} h\left(\frac{t+k \pi}{n}\right)(-1)^{k} \frac{\sin t}{t+k \pi} d t .
$$

For even $N$, the absolute value of the integrand here is dominated by

$$
\left|\sum_{1}^{N-1}\left[h\left(\frac{t+k \pi}{n}\right) \frac{1}{t+k \pi}-h\left(\frac{t+(k+1) \pi}{n}\right) \frac{1}{t+(k+1) \pi}\right]\right| \text {, }
$$

where ${ }^{*}$ again indicates summation over odd indices. If $N$ is odd, then

$$
\int_{N \pi / n}^{(N+1) \pi / n} h(t) \frac{\sin n t}{t} d t=o(1)
$$

just as $I_{2}$ did, and, by removing this term, we reduce the problem to one in which the sum has an even number of terms. We shall therefore assume $N$ to be even. The general term of the sum under consideration equals

$$
\begin{aligned}
{\left[h\left(\frac{t+k \pi}{n}\right)\right.} & \left.-h\left(\frac{t+(k+1) \pi}{n}\right)\right] \frac{1}{t+k \pi} \\
& -h\left(\frac{t+(k+1) \pi}{n}\right)\left[\frac{1}{t+k \pi}-\frac{1}{t+(k+1) \pi}\right] .
\end{aligned}
$$

Given $\epsilon>$ and choosing $N_{0}$ such that $\sum_{N_{0}+1}^{\infty} 1 / k^{2}<\epsilon$, we have

$$
\begin{aligned}
& \left|\sum_{1}^{N-1} h\left(\frac{t+(k+1) \pi}{n}\right)\left[\frac{1}{t+k \pi}-\frac{1}{t+(k+1) \pi}\right]\right| \\
& \leqslant \sum_{1}^{N-1}\left|f\left(x+\frac{t+(k+1) \pi}{n}\right)-f(x+0)\right| / k^{2}=\sum_{1}^{*}+\sum_{N_{0}+1}^{N-1}
\end{aligned}
$$


and the second sum is bounded by $2 \epsilon \sup |f(x)|$.The first sum is bounded by

$$
\sup _{0<t<\left(N_{0}+2\right) \pi / n}|f(x+t)-f(x+0)| \cdot \sum_{1}^{N_{0}} 1 / k^{2}
$$

which is $o(1)$ as $n \rightarrow \infty$ for each $x$, is $o(1)$ uniformly in $x$ in any closed interval of points of continuity, and is bounded uniformly in $n$ and $x$.

Finally we have

$$
\begin{aligned}
& \left|\sum_{1}^{N-1}\left[h\left(\frac{t+k \pi}{n}\right)-h\left(\frac{t+(k+1) \pi}{n}\right)\right] \frac{1}{t+k \pi}\right| \\
& \leqslant \sum_{1}^{N-1}\left|f\left(x+\frac{t+k \pi}{n}\right)-f\left(x+\frac{t+(k+1) \pi}{n}\right)\right| / k \leqslant V_{H}(\bar{f} ;[x, x+\delta]),
\end{aligned}
$$

where $\bar{f}(t)$ is $f(t)$ on $(x, x+\delta]$ and $\bar{f}(x)=f(x+0)$. Now

$$
V_{H}(\bar{f} ;[x, x+\delta])<\epsilon
$$

if $\delta$ is sufficiently small, since $\bar{f}$ is continuous on the right at $x$ [3]. If $f$ is continuous of each point of a closed interval $I$, then we may choose $\delta>0$ such that $V_{H}(f ;[x, x+\delta])<\epsilon$ for every $x \in I$. Clearly the sum is bounded by $V_{H}(f ;[0,2 \pi])$ for every $n$ and $x$.

\section{REFERENCES}

1. D. Waterman, On convergence of Fourier series of functions of generalized bounded variation, Studia Math. 44 (1972), 107-117; errata, ibid. 44 (1972), 651.

2. On the summability of Fourier series of functions of $\Lambda$-bounded variation, Studia Math. 55 (1976), 87-95.

3. _ On $\Lambda$-bounded variation, Studia Math. 57 (1976), 33-45.

4. A. Zygmund, Trigonometric series, Vol. I, Cambridge Univ. Press, Cambridge, 1959.

Department of Mathematics, Syracuse University, Syracuse, New York 13210 